\title{
On Some Representations of the Anticommutations Relations
}

G. RIDEAU

Institut H.-Poincaré, Paris

Received April 20, 1968

Abstract. We study representations of the canonical anticommutation relations having the form:

$$
\begin{aligned}
A(f) & =a(H f)+b^{*}(K f) \\
A^{*}(f) & =a^{*}(H f)+b(K f)
\end{aligned}
$$

where $a(f), b^{*}(f)$ and their adjoints are two basic anticommuting fields in a Fock Space.

A complete determination of the type in terms of $|K|=\left(K^{*} K\right)^{1 / 2}$ and a sufficient condition for quasi-equivalence are given.

\section{Introduction}

Let $\mathcal{F}$ be a complex Hilbert space of test functions, denoted by $f, g, h, \ldots$ To each element $f$ of $\mathbb{E}$ correspond two bounded operators on a Hilbert space $\mathfrak{F}, a(f)$ and $b^{*}(f)$, depending linearly and continuously on $f$ in the uniform topology of operators. We denote briefly their adjoints by $a^{*}(f)$ and $b(f)$; therefore, these are semi-linear in $f$. We impose the relations:

$$
\begin{aligned}
& {[a(f), a(g)]_{+}=\left[b^{*}(f), b^{*}(g)\right]_{+}=\left[a(f), b^{*}(g)\right]_{+}=[a(f), b(g)]_{+}=0} \\
& {\left[a(f), a^{*}(g)\right]_{+}=\left[b(g), b^{*}(f)\right]_{+}=(f, g)} \\
& f, g \in \mathfrak{G},[A, B]_{+}=A B+B A,
\end{aligned}
$$

and we take for $\mathfrak{F}$ the customary Fock-space associated with these two anticommutating fields. Id est, we have in $\mathfrak{F}$ a vector $\Omega_{0}$ such that:

$$
a(f) \Omega_{0}=b(g) \Omega_{0}=0, \quad f, g \in \mathfrak{F}
$$

and all the linear combinations of vectors having the form:

$$
a^{*}\left(f_{1}\right) \ldots a^{*}\left(f_{m}\right) b^{*}\left(g_{1}\right) \ldots b^{*}\left(g_{n}\right) \Omega_{0}
$$

are a dense set in $\mathfrak{F}$.

Now, if $H$ and $K$ are operators in $\mathfrak{E}(\mathbb{E})$ which satisfy:

$$
H^{*} H+K^{*} K=I
$$

we set:

$$
A(f)=a(H f)+b^{*}(K f) .
$$


Clearly, $A(f)$ is linear and norm-continuous in $f$. Moreover, if $A^{*}(f)$ is the adjoint of $A(f)$, a simple calculation gives:

$$
[A(f), A(g)]_{+}=0, \quad\left[A(f), A^{*}(g)\right]_{+}=(f, g) .
$$

Thus, we have defined by (4) a representation of the canonical anticommutation relations (CAR in the following). These representations have been introduced in [1] and are useful for describing gauge invariant generalized free fermion field [2], in particular, a free fermion gas with constant density at finite temperature [3]. Their study mainly from a mathematical point of view, is the purpose of this paper.

First, we recall some facts about the CAR. The most out-standing is the existence of a canonical $C^{*}$-algebra $\mathfrak{A}$ which can be viewed as generated by the $A(f)$ 's and their adjoints. Detailed constructions of it can be found in [4]. $\mathfrak{A}$ is a uniformly hyperfinite $C^{*}$-algebra [5].

With the concept of $C^{*}$-algebra is associated the concept of state: a state $\omega$ is a positive linear functional on the $C^{*}$-algebra with norm one [6]. In our case, a state $\omega$ is uniquely determined by the quantities:

$$
\omega\left(A^{*}\left(f_{1}\right) \ldots A^{*}\left(f_{n}\right) A\left(g_{1}\right) \ldots A\left(g_{m}\right)\right)
$$

that is, if we have, for two states $\omega_{1}$ and $\omega_{2}$ :

$$
\begin{aligned}
& \omega_{1}\left(A^{*}\left(f_{1}\right) \ldots A^{*}\left(f_{n}\right) A\left(g_{1}\right) \ldots A\left(g_{m}\right)\right) \\
= & \omega_{2}\left(A^{*}\left(f_{1}\right) \ldots A^{*}\left(f_{n}\right) A\left(g_{1}\right) \ldots A\left(g_{m}\right)\right)
\end{aligned}
$$

for all $f_{i}$ and $g_{j}$ in $\mathfrak{F}$, these states are identical.

A representation $\pi$ of the $C^{*}$-algebra $\mathfrak{A}$ defined in the Hilbert space $H_{\pi}$ is cyclic if there exits in $H_{\pi}$ a vector $\Omega$ such that the set of vectors $\{\pi(x) \Omega, x \in \mathfrak{U}\}$ is a total one in $H_{\pi}$. If $\Omega$ is normed to one, the quantity:

$$
\omega(x)=(\pi(x) \Omega, \Omega), \quad x \in \mathfrak{U}
$$

defines a state on $\mathfrak{A}$. Conversely, to each state on $\mathfrak{A}$ can be associated canonically a cyclic representation. We have the evident result of which we shall make use in the following:

If the same state is ascribed to distinct cyclic representations these representations are equivalent.

An important notion, which is basic for our work, is the quasiequivalence of two representations [6]. Among many definitions, we take the following:

Two representations $\pi_{1}$ and $\pi_{2}$ of a $C^{*}$-algebra are quasi-equivalent if there exist a multiple of $\pi_{1}$ and a multiple of $\pi_{2}$ which are equivalent.

It should be noticed that the quasi-equivalence is a true equivalence relation. 


\section{Some Auxilary Results on Quasi-Equivalence}

Let $V_{H}$ and $V_{K}$ closed subspaces of $\mathbb{E}$ spanned by the values of $H$ and $K$ :

$$
V_{H}=\overline{\{H f, f \in \mathbb{E}\}} \quad V_{K}=\overline{\{K f, f \in \mathbb{F}\}}
$$

and $V_{H}^{\frac{1}{H}}, V_{K}^{\frac{1}{K}}$ be their complementary subspaces. Generally, $V_{H}^{\frac{1}{H}}$ and $V_{\frac{1}{K}}^{\frac{1}{2}}$ are distincts from the null space. We denote by $h_{i}, i=1,2, \ldots$ and $k_{i}$, $i=1,2, \ldots$ some orthonormal basis in each of them. Now let $\mathfrak{F}_{i_{1}}^{H} \cdots i_{p} ; j_{1} \cdots j_{q}$ be the closed subspace of $\mathfrak{F}$ spanned by the vectors:

$$
\begin{gathered}
A^{*}\left(f_{1}\right) \ldots A^{*}\left(f_{n}\right) A\left(g_{1}\right) \ldots A\left(g_{m}\right) a^{*}\left(h_{i_{1}}\right) \ldots a^{*}\left(h_{i_{p}}\right) b^{*}\left(k_{j_{1}}\right) \ldots b^{*}\left(k_{j_{q}}\right) \Omega_{0} \\
n=0,1,2, \ldots ; \quad m=0,1,2, \ldots ; \quad f_{i} \in \mathbb{E} ; \quad g_{j} \in \mathbb{E} .
\end{gathered}
$$

One the one hand, $\mathfrak{F}_{i_{1} \ldots i_{p} ; j_{1} \ldots j_{q}}^{H K}$ is an invariant subspace for the representation (4) in which this representation is restricted to a cyclic representation with $a^{*}\left(h_{i_{1}}\right) \ldots a^{*}\left(h_{i_{p}}\right) b^{*}\left(k_{i_{1}}\right) \ldots b^{*}\left(k_{i_{p}}\right) \Omega_{0}$ as cyclic vector. These subrepresentations are all equivalent because the states generated on the $C^{*}$-algebra of the CAR by the various cyclic vector are identical. This results almost immediately from the anticommutation of the $A(f)$ 's and $A^{*}(f)$ 's with the $a^{*}\left(h_{i}\right)$ 's and $b^{*}\left(k_{j}\right)$ 's.

On the other hand, we have:

$$
\mathfrak{F}=\bigoplus_{p, q} \underset{\substack{i_{1} \ldots i_{p} \\ j_{1} \ldots j_{q}}}{\bigoplus} \mathfrak{F}_{i_{1} \ldots i_{p} ; j_{1} \ldots j_{q}}^{H} .
$$

Indeed, it can be proved easily by induction that each vector in $\mathfrak{F}$ having the form:

$a^{*}\left(H f_{1}\right) \ldots a^{*}\left(H f_{n}\right) b^{*}\left(K g_{1}\right) \ldots b^{*}\left(K g_{n}\right) a^{*}\left(h_{i_{1}}\right) \ldots a^{*}\left(h_{i_{p}}\right) b^{*}\left(k_{j_{1}}\right) \ldots b^{*}\left(k_{j_{q}}\right) \Omega_{0}$

can be written as a linear combination of vectors having the form (6). Let now, in (7), $f_{1}, \ldots, f_{n}, g_{1}, \ldots, g_{m}$ run over $\leftleftarrows, p$ and $q$ run over all integers and $i_{1} \ldots i_{p}$ and $j_{1} \ldots j_{q}$ over all choice of the indices, we obtain a set of vectors which is a total one in $\mathfrak{F}$; then we get:

Theorem 1. The representation of the CAR defined by (4) is a multiple of a cyclic representation. The multiplicity is equal to $2^{r+s}$, where $r$ and $s$ are the dimensions of $V_{\vec{H}}^{\frac{1}{H}}$ and $V_{\bar{K}} \frac{1}{\text {. }}$

In our case, the state which defines the cyclic representation satisfy:

$$
\begin{aligned}
\omega\left(A\left(f_{1}\right) \ldots A\left(f_{n}\right) A\left(g_{1}\right) \ldots A\left(g_{m}\right)\right) & =\left(A^{*}\left(f_{1}\right) \ldots A^{*}\left(f_{n}\right) A\left(g_{1}\right) \ldots\right. \\
\left.\ldots A\left(g_{m}\right) \Omega_{0}, \Omega_{0}\right) & =(-1)^{\frac{n(n-1)}{2}} \delta_{n m} \operatorname{det}\left(K^{*} K g_{i}, f_{j}\right) .
\end{aligned}
$$

Since the knowledge of these quantities characterizes completely the state, it is clear that two representations (4) with the same value of $K^{*} K$ can differ only by their multiplicity, and then are quasi-equivalent. 
Corollary. All the representations (4) having the same value of $|K|=\left(K^{*} K\right)^{1 / 2}$ are quasi-equivalent.

In particular, the representation (4) is quasi-equivalent to the representation defined by:

$$
A(f)=a(|H| f)+b^{*}(|K| f) .
$$

Lemma 1. If $H$ and $K$ in $\mathfrak{L}(\mathfrak{F})$ satisfy the relations:

$$
\begin{array}{ll}
H^{*} H+K^{*} K=I & H H^{*}+K K^{*}=I \\
H^{*} K=K^{*} H & H K^{*}=K H^{*}
\end{array}
$$

and if $K$ is an Hilbert-Schmidt operator, there exits in $\mathfrak{I}(\mathfrak{F})$ a unitary operator $U$ such that:

$$
\left.\begin{array}{c}
A(f)=a(H f)+b^{*}(K f)=U a(f) U^{*} \\
B^{*}(f)=-a(K f)+b^{*}(H f)=U b(f) U^{*} .
\end{array}\right\}
$$

Proof. The representation of two anticommuting fields defined by the right member in (9) is irreducible. Indeed, from (8), we get:

$$
\begin{aligned}
& a(f)=A\left(H^{*} f\right)-B^{*}\left(K^{*} f\right) \\
& b^{*}(f)=A\left(K^{*} f\right)+B\left(H^{*} f\right) .
\end{aligned}
$$

Therefore, an operator commuting with $A(f), B^{*}(f)$ commutes with $a(f), b^{*}(f)$ and thus is scalar.

Now, if $K$ is an Hilbert-Schmidt operator, it can be written [7]:

$$
K f=\sum_{i} \lambda_{i} g_{i}\left(f, f_{i}\right), \quad \lambda_{i} \geqq 0 \quad \Sigma \lambda_{i}^{2}<\infty,
$$

where $\left\{f_{i}, i=1,2, \ldots\right\}$ is an orthonormal basis and $\left\{g_{i}, i=1,2, \ldots\right\}$ an orthonormal set of vectors in $\mathcal{E}$. The $\lambda_{i}$ 's are eigenvalues of $|K|$. By (8), we have for $H$ :

$$
H f=\sum_{i} \sqrt{1-\lambda_{i}^{2}} h_{i}\left(f, f_{i}\right),
$$

where $\left\{h_{i}, i=1,2, \ldots\right\}$ is an orthonormal set of vectors. Moreover, by the first Eq. (8), we have:

$$
\lambda_{i}^{2}\left(h_{i}, g_{j}\right)=\lambda_{j}^{2}\left(h_{i}, g_{j}\right)
$$

So, $\left(h_{i}, g_{j}\right)=0$ if $\lambda_{i} \neq \lambda_{j}$. But for $\lambda_{i} \neq 0, \lambda_{i}=\lambda_{j}$ is possible only for a finite number of $j$, since $K$ is Hilbert-Schmidt. Let $i_{1}, i_{2}, \ldots i_{n}$ be these values of $j$. From the second Eq. (8), we get:

$$
\left(g_{i_{k}}, h_{i_{l}}\right)=\left(h_{i_{k}}, g_{i_{\ell}}\right)
$$

Hence the matrix with elements $\left(g_{i_{k}}, h_{i_{l}}\right)$ is unitary and hermitian. So, its eigenvalues are \pm 1 and there exists a unitary finite dimensional matrix $u$, such that:

$$
\sum_{k} h_{i_{k}} u_{k l}= \pm \sum_{k} g_{i_{k}} u_{k l}
$$


Setting :

we have:

$$
g_{i l}^{\prime}=\sum_{k} g_{i_{k}} u_{k l}
$$

$$
g_{i_{k}}=\sum_{l} \bar{u}_{k l} g_{i_{l}}^{\prime}, h_{i_{k}}=\sum_{l} \bar{u}_{k l} \varepsilon_{1} g_{i l}^{\prime}, \quad \varepsilon_{l}= \pm 1
$$

and the terms $\lambda_{i} \sum_{k} g_{i_{k}}\left(f, f_{i_{k}}\right), \sqrt{1-\lambda_{i}^{2}} \sum_{k} h_{i_{k}}\left(f, f_{i_{k}}\right)$ in the expansions of $H f$ and $K f$ become respectively:

$$
\lambda_{i} \sum_{k} g_{i_{k}}^{\prime}\left(f, \sum_{l} f_{i_{l}} u_{l k}\right), \quad \sqrt{1-\lambda_{i}^{2}} \sum_{k} \varepsilon_{k} g_{i_{k}}^{\prime}\left(f, \sum_{l} f_{i l} u_{l k}\right) \quad \varepsilon_{k}= \pm 1 .
$$

If $\lambda_{i}$ is zero, the corresponding $f_{i}$ and $g_{i}$ in the expansion of $K$ are immaterial and we can always assume $h_{i}=g_{i}$ for all $i$ with $\lambda_{i}=0$, so that, setting now:

$$
f_{i_{k}}^{\prime}=\sum_{l} f_{i l} u_{l k}
$$

and dropping the dashes, we can write for $H f$ and $K f$ :

$$
K f=\sum_{i} \lambda_{i} g_{i}\left(f, f_{i}\right), \quad H f=\sum_{i} \sqrt{1-\lambda_{i}^{2}} \varepsilon_{i} g_{i}\left(f, f_{i}\right) \quad \varepsilon_{i}= \pm 1
$$

where, this time, $g_{i}, i=1,2, \ldots$ is an orthonormal basis in $F$, as implied by $H H^{*}+K K^{*}=I$.

Then, we have:

$$
\begin{array}{ll}
A(f)=\sum_{i}\left(f, f_{i}\right)\left(\sqrt{1-\lambda_{i}^{2}} \varepsilon_{i} a\left(g_{i}\right)+\lambda_{i} b^{*}\left(g_{i}\right)\right) & \\
B(f)=\sum_{i}\left(f, f_{i}\right)\left(-\lambda_{i} a^{*}\left(g_{i}\right)+\sqrt{1-\lambda_{i}^{2}} \varepsilon_{i} b\left(g_{i}\right)\right) . & \varepsilon_{i}= \pm 1
\end{array}
$$

Now the vector:

$$
\Omega=\Pi_{i}\left(\sqrt{1-\lambda_{i}^{2}}-\varepsilon_{i} \lambda_{i} a^{*}\left(g_{i}\right) b^{*}\left(g_{i}\right)\right) \Omega_{0}
$$

is in $\mathfrak{F}$, as follows from the condition $\sum_{i} \lambda_{i}^{2}<\infty$, and a straightforward calculation gives:

$$
A(f) \Omega=B(g) \Omega=0, \quad f, g \in \mathfrak{E} ;
$$

Thus, the $A(f), B^{*}(g)$ defined in (9) determine an irreducible Fock representation in the same way as the $a(f)^{\prime}$ s and $b^{*}(g)^{\prime}$ 's, therefore these two representations are unitarily equivalent. This completes the proof of the lemma.

As a first application, we can derive the:

Lemma 2. If, in (4), $H$ and $K$ are positive operators, we can always assume that $K$ does not admit zero or one as eigenvalues.

Proof. Let $\left\{f_{i}, i=1,2, \ldots\right\}$ and $\left\{g_{j}, j=1,2, \ldots\right\}$ be orthonormal basis in the eigenspaces of $\mathbb{F}$ corresponding to the eigenvalues one and 16 Commun. math. Phys., Vol. 9 
zero respectively. For $f$ in $\mathfrak{~}$, we may write:

$$
f=\sum_{i} \alpha_{i} f_{i}+\sum_{j} \beta_{j} g_{j}+h, \quad\left(h, f_{i}\right)=\left(h, g_{j}\right)=0, i, j=1,2, \ldots
$$

Moreover, by (3):

$$
H f_{i}=0, \quad i=1,2, \ldots, H g_{j}=g_{j} \quad i=1,2, \ldots
$$

Now, let $\left\{\theta_{i}, i=1,2, \ldots\right\}$ and $\left\{\omega_{j}, j=1,2, \ldots\right\}$ be two sets of arguments such that:

$$
\begin{gathered}
0<\theta_{i}<\frac{\pi}{2}, \quad 0<\omega_{j}<\frac{\pi}{2} \\
\sum \theta_{i}^{2}<\infty, \quad \sum \omega_{j}^{2}<\infty .
\end{gathered}
$$

From the preceding lemma, we deduce the existence of an unitary operator $V$ in $\mathfrak{L}(\mathfrak{F})$ such that:

$$
\begin{aligned}
V a(f) U^{*}= & \sum_{i} \alpha_{i}\left(\cos \theta_{i} a\left(f_{i}\right)+\sin \theta_{i} b^{*}\left(f_{i}\right)\right) \\
& +\sum_{j} \beta_{j}\left(\cos \omega_{j} a\left(g_{j}\right)+\sin \omega_{j} b^{*}\left(g_{j}\right)\right)+a(h), \\
U b^{*}(f) U^{*}= & \sum_{i} \alpha_{i}\left(-\sin \theta_{i} a\left(f_{i}\right)+\cos \theta_{i} b^{*}\left(f_{i}\right)\right) \\
& +\sum_{j} \beta_{j}\left(-\sin \omega_{j} a\left(g_{j}\right)+\cos \omega_{j} b^{*}\left(g_{j}\right)\right)+b^{*}(h) .
\end{aligned}
$$

Since the space spanned by the vectors $h$ of (10) is invariant by $H$ and $K$, we have:

$$
\begin{aligned}
A^{\prime}(f)= & U A(f) U^{*}=a(H h)+b(K h)+\sum_{i} \alpha_{i}\left(\cos \theta_{i} b^{*}\left(f_{i}\right)-\sin \theta_{i} a\left(f_{i}\right)\right) \\
& +\sum_{j} \beta_{j}\left(\cos \omega_{j} a\left(g_{j}\right)+\sin \omega_{j} b^{*}\left(g_{j}\right)\right)=a\left(H^{\prime} f\right)+b^{*}\left(K^{\prime} f\right)
\end{aligned}
$$

with:

$$
\begin{aligned}
& K^{\prime} f=K f-\sum_{i}\left(1-\cos \theta_{i}\right)\left(f, f_{i}\right) f_{i}+\sum_{j} \sin \omega_{j}\left(f, g_{j}\right) g_{j} \\
& H^{\prime} f=H f-\sum_{i} \sin \theta_{i}\left(f, f_{i}\right) f_{i}-\sum_{j}\left(1-\cos \omega_{j}\right)\left(f, g_{j}\right) g_{j} .
\end{aligned}
$$

If $f$ given by (10) must verify $K^{\prime} f=0$, we have:

$$
K h+\sum_{i} \alpha_{i} \cos \theta_{i} f_{i} \sum_{j} \beta_{j} \sin \omega_{j} g_{j}=0 .
$$

The inequalities imposed on $\theta_{i}$ and $\omega_{j}$ imply $\alpha_{i}=\beta_{j}=0$; but, from $K h=0$, follows $h=0$ by the definition of $h$. In the same way, we shall show that $K^{\prime} f=f$ implies $f=0$. Thus we have constructed a representation with the required property, unitarily equivalent to the initial one.

Remark. One can show easily that in the conditions of the lemma, the representation is cyclic with $\Omega_{0}$ as cyclic vector. This remark shall be of some use in the following (cf. section III). 
Let us now consider two representations of the type (4):

$$
\begin{aligned}
& A_{1}(f)=a\left(H_{1} f\right)+b\left(K_{1} f\right), \\
& A_{2}(f)=a\left(H_{2} f\right)+b\left(K_{2} f\right),
\end{aligned}
$$

where $H_{1}, K_{1}$ and $H_{2}, K_{2}$ are assumed positive. We state:

Theorem 2. If $\left(H_{1} K_{2}-K_{1} H_{2}\right)$ is an Hilbert-Schmidt operator, the representations (12) and (13) are unitarily equivalent.

Proof. Let us consider:

$$
\begin{aligned}
& a^{\prime}(f)=a\left(\left(H_{1} H_{2}+K_{1} K_{2}\right) f\right)-b^{*}\left(\left(H_{1} K_{2}-K_{1} H_{2}\right) f\right), \\
& b^{\prime}(f)=a\left(\left(H_{1} K_{2}-K_{1} H_{2}\right) f\right)+b^{*}\left(\left(H_{1} H_{2}+K_{1} K_{2}\right) f\right) .
\end{aligned}
$$

Since $\left[H_{1}, K_{1}\right]=\left[H_{2}, K_{2}\right]=0$, the operators $H=H_{1} H_{2}+K_{1} K_{2}$ and $K=K_{1} H_{2}-H_{1} K_{2}$ have the properties stated in the lemma 1. Therefore there exists a unitary operator $U$ in $\mathfrak{L}(\mathfrak{F})$ with:

But:

$$
a^{\prime}(f)=U a(f) U^{*}, \quad b^{\prime}(f)=U b(f) U^{*} .
$$

Hence :

$$
a^{\prime}\left(H_{2} f\right)+b^{*}\left(K_{2} f\right)=a\left(H_{1} f\right)+b^{*}\left(K_{1} f\right)=A_{1}(f)
$$

$$
A_{1}(f)=U A_{2}(f) U^{*}
$$

\section{The Main Results}

Since, by (3), $K$ is bounded by one, we can define an operator $\Theta$ by:

$$
\Theta=\operatorname{Arcsin}|K| \text {. }
$$

Theorem 3. Each representation (4) is quasi-equivalent to a similar representation for which the corresponding $|K|$ has a discrete spectrum.

Proof. Let us assume $\Theta$ have a spectrum with a continuous part. von Neumann has shown [8] that we can add to $\Theta$ a Hilbert-Schmidt operator, $A$ such that $\Theta+A$ has a discrete spectrum contained in $[0, \pi / 2]$ as $\Theta$ spectrum is ${ }^{1}$. Moreover, $A$ can be chosen so that $\|A\|<\varepsilon$, where $\varepsilon$ is some positive number. We can form $\cos (\Theta+A)$ and $\sin (\Theta+A)$ which are positive operators and consider the representation defined by:

$$
A^{\prime}(f)=a(\cos (\Theta+A) f)+b(\sin (\Theta+A) f) .
$$

Using a technical device customary in quantum field theory, we have:

$$
\begin{aligned}
e^{i(\Theta+A)=} & e^{i \Theta}\left(1+i \int_{0}^{1} A(t) d t+i^{2} \int_{0}^{1} d t_{1} \int_{0}^{t_{1}} d t_{2} A\left(t_{1}\right) A\left(t_{2}\right)\right. \\
& \left.+\cdots+i \int_{0}^{1} d t_{1} \int_{0}^{t_{1}} d t_{2} \ldots \int_{0}^{t_{n-1}} d t_{n} A\left(t_{1}\right) A\left(t_{2}\right) \ldots A\left(t_{n}\right)+.\right)
\end{aligned}
$$

1 We owe this remark to DR. O. E. Lanford. 
Here $A(t)=e^{-i t \Theta} A e^{i t \Theta}$. Since $A(t)$ is a Hilbert-Schmidt operator with the same Hilbert-Schmidt norm as $A$, the series in the right member, minus his first term, converges in the Hilbert-Schmidt norm and defines an Hilbert-Schmidt operator. Thus we have:

$$
e^{i(\Theta+A)}=e^{i \Theta}+T,
$$

where $T$ is a Hilbert-Schmidt operator. Taking the adjoints of the two members, we get:

$$
e^{-i(\Theta+A)}=e^{-i \Theta}+T
$$

and these relations imply:

$$
\cos (\Theta+A)=\cos \Theta+\frac{T+T^{*}}{2}, \quad \sin (\Theta+A)=\sin \Theta+\frac{T-T^{*}}{2 i} .
$$

Now :

$$
\sin (\Theta+A) \cos \Theta-\cos (\Theta+A) \sin \Theta=\frac{1}{2}\left(T e^{i \Theta}+T^{*} e^{-i \Theta}\right)
$$

Since the right member is a Hilbert-Schmidt operator, it results from the lemma 2 that the representation (14) is equivalent unitarily to the representation defined by:

$$
A^{\prime \prime}(f)=a(\cos \Theta f)+b^{*}(\sin \Theta f)=a(|H| f)+b^{*}(|K| f) .
$$

But, as a by-product of the theorem 1, this last representation is quasiequivalent to the original one.

Therefore, if we do not make any distinction between quasi-equivalent representations, we may assume that $K$ in (4) is a positive operator with a complete discrete spectrum. Moreover, if we take in account the lemma 2, we may even assume that $K$ does not admit zero or one as eigenvalues. Thus we write:

$$
K=\sum_{i} \sin \theta_{i} P_{f_{i}}, \quad 0<\theta_{i}<\pi / 2
$$

where $P_{f_{i}}$ is the projection operator on the eigenvector $f_{i}$. Since the orthonormal set $f_{i}, i=1,2, \ldots$ is complete and $\Omega_{0}$ is a cyclic vector, we may characterize the representation by the set of numbers:

$$
\begin{aligned}
& \left(A^{*}\left(f_{i_{1}}\right) \ldots A^{*}\left(f_{i_{n}}\right) A\left(f_{j_{1}}\right) \ldots A\left(f_{j_{m}}\right) \Omega_{0}, \Omega_{0}\right) \\
= & \omega\left(A^{*}\left(f_{i_{1}}\right) \ldots A^{*}\left(f_{i_{n}}\right) A\left(f_{j_{1}}\right) \ldots A\left(f_{j_{m}}\right)\right),
\end{aligned}
$$

which define uniquely a state $\omega$ on the $C^{*}$-algebra of the CAR. All these numbers are zero, except if the sets $\left(i_{1}, \ldots i_{n}\right)$ and $\left(j_{1}, \ldots j_{m}\right)$ are identical up to the order. By the CAR, these last quantities can be written as linear combinations of $\omega\left(N\left(f_{i_{1}}\right) \ldots N\left(f_{i_{n}}\right)\right)$ where $N\left(f_{i}\right)=A^{*}\left(f_{i}\right) A\left(f_{i}\right)$, and a direct calculation gives:

$$
\omega\left(N\left(f_{i_{1}}\right) \ldots N\left(f_{i_{n}}\right)\right)=\prod_{k=1}^{n} \sin ^{2} \theta_{i_{k}} .
$$


We must remark that the adjunction of an Hilbert-Schmidt operator to a symetric operator does not change the limit points of the spectrum.

Thus if $\Theta$ has a continuous spectrum, the discrete spectrum of $\Theta+A$ admits all the points of a segment as accumulation points. This remark shall be used in the following.

\section{An Equivalent Representation}

We recall briefly in this section the construction of a family of representations of the CAR, the detailed study of which was made in $[9,10]$ in a slightly different form. To each $i, i=1,2, \ldots$, let us correspond two two-dimensional Hilbert-spaces $\mathscr{H}_{i}^{(1)}$ and $\mathscr{H}_{i}^{(2)}$ and let be $\mathscr{H}_{i}=\mathscr{H}_{i}^{(1)} \otimes \mathscr{H}_{i}^{(2)}$. If $\theta_{i}, i=1,2, \ldots$, is a set of arguments verifying the inequalities $0<\theta_{i}<\pi / 2$, we denote by $I_{1}$ the set of indices such that $0<\theta_{i} \leqq \pi / 4$ and by $I_{2}$ the set of indices such that $\pi / 4<\theta_{i}<\pi / 2$. We choose in each $\mathscr{H}_{i}^{(\tau)}$ the basis vectors $e_{i j}^{(\tau)}, j=1,2$, and we consider $f_{i}$ in $\mathscr{H}_{i}$ given by:

or :

$$
f_{i}=\cos \theta_{i} e_{i, 1}^{(1)} \otimes e_{i, 1}^{(2)}+\sin \theta_{i} e_{i, 2}^{(1)} \otimes e_{i, 2}^{(2)} \quad \text { for } \quad i \in I_{1}
$$

$$
f_{i}=\sin \theta_{i} e_{i, 1}^{(1)} \otimes e_{i, 1}^{(2)}+\cos \theta_{i} e_{i, 2}^{(1)} \otimes e_{i, 2}^{(2)} \quad \text { for } \quad i \in I_{2} .
$$

Let us form the incomplete direct product $\mathfrak{G}$ of the $\mathscr{H}_{i}$ generated by the $f_{i}$ 's $[11]$ :

$$
\mathfrak{G}=\stackrel{f}{i}^{\mathscr{H}_{i}} \mathscr{H}_{i} .
$$

If $a_{i}, a_{i}^{*}$ are operators in $\mathscr{H}_{i}^{(1) 2}$ verifying:

or:

$$
\begin{array}{ll}
a_{i} e_{i, 1}^{(1)}=0 & a_{i}^{*} e_{i, 1}^{(1)}=e_{i, 2}^{(1)} \\
a_{i} e_{i, 2}^{(1)}=0 & a_{i}^{*} e_{i, 2}^{(1)}=0 \quad \text { for } \quad i \in I_{1}
\end{array}
$$

$$
\begin{array}{ll}
a_{i} e_{i, 1}^{(1)}=e_{i, 2}^{(1)} & a_{i}^{*} e_{i, 1}^{(1)}=0 \\
a_{i} e_{i, 2}^{(1)}=0 & a_{i}^{*} e_{i, 1}^{(1)}=e_{i, 2}^{(1)} \quad \text { for } \quad i \in I_{2}
\end{array}
$$

we obtain a representation of the CAR in $\mathfrak{L}(\mathfrak{G})$ by setting:

$$
A_{i}=\prod_{j<i}\left(1-2 a_{j}^{*} a_{j}\right) a_{i}, \quad A_{i}^{*}=\prod_{j<i}\left(1-2 a_{j}^{*} a_{j}\right) a_{i}^{*},
$$

where we have adopted the same notations for $a_{i}$ and $a_{i}^{*}$ in $\mathcal{L}\left(\mathscr{H}_{i}^{(1)}\right)$ and their extension to $\mathfrak{L}(\mathfrak{G})$.

The assumption on the $\theta_{i}$ 's implies the cyclicity of $\otimes f_{i}$ and an easy but tedious calculation shows that the corresponding state is identical with the state of the preceding section. Since a cyclic representation of a $C^{*}$-algebra is characterized up to an equivalence by its defining state, we get the theorem:

${ }^{2}$ These $a_{i}, a_{i}^{*}$ must not be confused with the $a(f), a^{*}(f)$ of the preceding section. 
Theorem 4. For each representation of the CAR defined by (4), there is a set of $\theta_{i}, i=1,2, \ldots$ with $0<\theta_{i}<\pi / 2$ and a complete orthonormal basis in $\mathfrak{F},\left\{f_{i}, i=1,2, \ldots\right\}$ such that the representation is equivalent to the product representation defined by (15) where $A_{i}=A\left(f_{i}\right)$.

The representations (15) can be completely classified according to their types $[12,14]$. We quote here the statements of [14], adapted to our particular case:

1) The representations are of type $I_{\infty}$ if, and only if:

$$
\sum_{i \in I_{1}}\left(1-\cos ^{2} \theta_{i}\right)+\sum_{i \in I_{2}}\left(1-\sin ^{2} \theta_{i}\right)<\infty .
$$

2) The representations are of type $I I_{1}$ if, and only if:

$$
\sum_{i \in I}\left(1-\frac{1}{\sqrt{2}}\left(\cos \theta_{i}+\sin \theta_{i}\right)\right)<\infty
$$

3) The representations are of type III if, and only if, for some $c>0$ :

$$
\sum_{i \in I_{1}} \sin ^{2} \theta_{i}\left|\frac{\cos ^{2} \theta_{i}}{\sin ^{2} \theta_{i}}-1\right|_{c}^{2}+\sum_{i \in I_{2}} \cos ^{2} \theta_{i}\left|\frac{\sin ^{2} \theta_{i}}{\cos ^{2} \theta_{i}}-1\right|_{c}^{2}=\infty .
$$

where $|x|_{c}=\inf (|x|, c)$.

4) In all other cases, the representations are of type $I I_{\infty}$.

Let us now give the detailed consequences for $|K|$. In case (1), we denote by $E$ the orthogonal projection on the subspace spanned by the vectors $f_{i}, i \in I_{1}$. Since inequality (16) is equivalent to:

$$
\sum_{i \in I_{1}} \theta_{i}^{2}+\sum_{i \in I_{2}}\left(\frac{\pi}{2}-\theta_{i}\right)^{2}<\infty
$$

if we take into account $(\Theta+A) f_{i}=\theta_{i} f_{i}$, we deduce:

$$
\left(\Theta+A-\frac{\pi}{2}\right)(1-E)+(\Theta+A) E=\text { Hilbert-Schmidt operator. }
$$

But $A$ is also a Hilbert-Schmidt operator. Then:

$$
\Theta=\frac{\pi}{2}(1-E)+\text { Hilbert-Schmidt operator. }
$$

Hence:

$$
|K|=\sin \Theta=1-E+\text { Hilbert-Schmidt operator. }
$$

Conversely, if $|K|$ has this form, the representation is of type $I_{\infty}$ because, from theorem 2 , it is quasi-equivalent to the representation corresponding to $|K|=1-E$, which is a discrete one in the sense of WightmanGarding [13].

Let us now consider the case (2). Inequality (17) is equivalent to:

$$
\sum_{i \in I}\left(\frac{\pi}{4}-\theta_{i}\right)^{2}<\infty
$$

which implies, since $A$ is Hilbert-Schmidt operator:

$$
\Theta=\frac{\pi}{4} I+\text { Hilbert-Schmidt operator }
$$


or, also:

$$
|K|=\frac{1}{\sqrt{2}} I+\text { Hilbert-Schmidt operator. }
$$

The discussion of the case (3) is a little more involved. Let us denote by $I_{1}^{1}, I_{1}^{2}$ the subsets of $I_{1}$ such that:

$$
\left(\frac{\cos ^{2} \theta_{i}}{\sin ^{2} \theta_{i}}-1\right)>c \quad \text { if } \quad i \in I_{1}^{1},\left(\frac{\cos ^{2} \theta_{i}}{\sin ^{2} \theta}-1\right)<c \quad \text { if } \quad i \in I_{1}^{2} .
$$

Similarly, let us denote by $I_{2}^{1}, I_{2}^{2}$, the subsets of $I_{2}$ such that:

$$
\left(\frac{\sin ^{2} \theta_{i}}{\cos ^{2} \theta_{i}}-1\right)<c \quad \text { if } \quad i \in I_{2}^{1},\left(\frac{\sin ^{2} \theta_{i}}{\cos ^{2} \theta_{i}}-1\right)>c \quad \text { if } \quad i \in I_{2}^{2} .
$$

Thus, (18) can be written:

$$
c^{2} \sum_{i \in I_{1}^{1}} \sin ^{2} \theta_{i}+\sum_{i \in I_{1}^{2}} \frac{\cos ^{2} 2 \theta_{i}}{\sin ^{2} \theta_{i}}+\sum_{i \in I_{2}^{1}} \frac{\cos ^{2} 2 \theta_{i}}{\cos ^{2} \theta_{i}}+c^{2} \sum_{i \in I_{2}^{2}} \cos ^{2} \theta_{i}=\infty .
$$

At least, one of the four series in the left member should be divergent. This is evidently the case if the $\theta_{i}$ 's have an accumulation point distinct of $0, \pi / 4$ and $\pi / 2$, or, equivalently, if $|K|$ spectrum has a limit point distinct of $0,1 / \sqrt{2}$ and 1 . In the opposite situation, we can always find three projection operators in $\mathfrak{F}, E_{1}, E_{2}, E_{3}$, commuting with $|K|$ and so that $|K| E_{1},|K| E_{2},|K| E_{3}$ must have the unique limit point $0,1 / \sqrt{2}$, and 1 respectively ${ }^{3}$. The corresponding representation is of type $I I I$ if, and only if, at least one of the $|K| E_{1},|K| E_{2}-\frac{1}{\sqrt{2}} E_{2},|K| E_{3}-E_{3}$ is compact but not Hilbert-Schmidt operator.

It is now very easy to characterize type $I I_{\infty}$ representations: $|K|$ spectrum possesses effectively the three limit points $0,1 / \sqrt{2}$ and 1 and the three operators $|K| E_{1},|K| E_{2}-\frac{1}{\sqrt{2}} E_{2},|K| E_{3}-E_{3}$ are all HilbertSchmidt operators ${ }^{4}$.

All this discussion can be summed up in the theorem:

Theorem 5. Let us consider the representation of the $C A R$ defined by (4). We state:

$\left.1^{\circ}\right)$ The representation is of type $I_{\infty}$ if, and only if, $|K|$ has the form:

$$
|K|=1-E+\text { Hilbert-Schmidt operator }
$$

where $E$ is some projection operator in $\mathfrak{L}(\mathbb{F})$.

$\left.2^{\circ}\right)$ The representation is to type $I I_{1}$ if, and only if, $|K|$ has the form:

$$
|K|=\frac{1}{\sqrt{2}} I+\text { Hilbert-Schmidt operator. }
$$

${ }^{3}$ It may be that one or two of the $E_{i}$ 's are the null operator.

${ }_{4}^{4}$ It may be that $E_{1}$ or $E_{3}$ is the null operator. 
$\left.3^{\circ}\right)$ The representation is of type $I I_{\infty}$ if, and only if, the spectrum of $K$ has the three limit points $0,1 / \sqrt{2}$ and 1 and the three operators $|K| E_{1}$, $|K| E_{2}-\frac{1}{\sqrt{2}} E_{2},|K| E_{3}-E_{3}$ are Hilbert-Schmidt operators, where $E_{1}, E_{2}$, $E_{3}$ are projection operators commuting with $|K|$ and chosen so that $|K| E_{1}$, $|K| E_{2},|K| E_{3}$ have spectra with the unique limit point $0,1 / \sqrt{2}$ and 1 respectively.

$\left.4^{\circ}\right)$ In all other cases, the representation is of type III.

In particular, if $|K|$ has a spectrum partly continuous, we have necessarily a limit point distinct of $0,1 / \sqrt{2}$ and 1 and consequently the representation is of type $I I I$. This is precisely the situation for the representation given in [3] and describing an infinite free fermion gas with constant density at a temperature $T$ which is finite and not zero.

\section{Complementary Results}

We can use theorem 5 for etablishing the reciprocal statement of the lemma 1 , and, to some extent, of the theorem 2 . Indeed, we have:

Lemma $1^{\prime}$. If $H$ and $K$ in $\mathfrak{I}(\mathbb{F})$ satisfy the relations (8) and if there exists $U$ unitary in $\mathfrak{I}(\mathfrak{F})$ such that relations $(9)$ are satisfied, $K$ is a HilbertSchmidt operator.

Proof. Since the representation of the CAR provided by the first line in (9) is of type $I_{\infty}$, it results from the first part of theorem 5 that:

$$
|K|=1-E+\text { Hilbert-Schmidt operator. }
$$

with $E$ some projection operator in $\mathfrak{L}(\mathfrak{F})$. Now, since this representation is equivalent to Fock-representation, it can be shown easily that $E$ is unity operator. Thus, $|K|$ is actually a Hilbert-Schmidt operator; and the same is evidently true for $K$.

Theorem 2'. Let us consider the two sets of operators:

$$
\left.\begin{array}{l}
A_{1}(f)=a\left(H_{1} f\right)+b^{*}\left(K_{1} f\right) \\
B_{1}^{*}(f)=-a\left(K_{1} f\right)+b^{*}\left(H_{1} f\right)
\end{array}\right\}
$$

where $H_{i}, K_{i}$ are positive and satisfy:

$$
H_{i}^{2}+K_{i}^{2}=I
$$

If there exists $U$ unitary in $\mathfrak{L}(\mathfrak{F})$ such that:

$$
A_{2}(f)=U A_{1}(f) U^{*}, \quad B_{1}^{*}(f)=U B_{1}^{*}(f) U^{*},
$$

the operator $H_{1} K_{2}-K_{1} H_{2}$ is necessarily Hilbert-Schmidt. 
Proof. We can write:

Hence:

$$
\begin{aligned}
& a(f)=A_{1}\left(H_{1} f\right)-B_{1}^{*}\left(K_{1} f\right) \\
& b^{*}(f)=A_{1}\left(K_{1} f\right)+B_{1}^{*}\left(H_{1} f\right) .
\end{aligned}
$$

$$
\begin{aligned}
U a(f) U^{*}=A_{2}\left(H_{1} f\right) & -B_{2}^{*}\left(K_{1} f\right)=a\left(\left(H_{2} H_{1}+K_{2} K_{1}\right) f\right) \\
& +b^{*}\left(\left(K_{2} H_{1}-H_{2} K_{1}\right) f\right) \\
U b^{*}(f) U^{*}=A_{2}\left(K_{1} f\right) & +B_{2}^{*}\left(K_{1} f\right)=-a\left(\left(K_{2} H_{1}-H_{2} K_{1}\right) f\right) \\
& +b^{*}\left(\left(H_{2} H_{1}+K_{2} K_{1}\right) f\right) .
\end{aligned}
$$

From the preceding lemma, $\left(K_{2} H_{1}-H_{2} K_{1}\right)$, hence its adjoint, is necessarily Hilbert-Schmidt.

Acknowledgements. We are indebted to Dr. R. T. Powers for many helpful discussions which have greatly contributed to improve this paper, to Pr. O. E. LaNFORD III, for valuable criticisms and suggestions, to Pr Stormer for having pointed out reference [14], and to R. WIGHTMAN and Pr. KADISON for their kind hospitality.

\title{
References
}

1. Shale, D., and H. W. F. Stinespring: Ann. Math. 80, 365 (1964).

2. Verbeure, A., and E. Baslev: I. H. E. S., preprints.

3. Araki, H., and W. Wyss: Helv. Phys. Acta 37, 136 (1964).

4. Powers, R. T.: Representation of the canonical anticommutation relations Princeton's Dissertation, june 1967.

Guichardet, A.: Ann. École Norm. Super. 83, 1 (1966).

5. Glimm, J.: Trans. Am. Math. Soc. 95, 318 (1960).

6. Dixmier, J.: Les $C$-algèbres et leurs représentations. Paris: Gauthier-Villars 1964.

7. Guelfand, I. M., et N. Y. Vilenkin: Les distributions, tome IV, Paris: Dunod 1967.

8. Neumann, J. von: Acta Sci. Ind. 229 (1935).

9. Cordesse, A., et G. Rideau : Nuovo Cimento 46 A, 624 (1966).

10. - - Nuovo Cimento III. 50 A, 244 (1967).

11. Neumann, J. von: Comp. Math. 6, 1 (1938).

12. J. D. C.: Bures Comp. Math. 15, 169 (1963).

13. Wightman, A., and L. GArding: Proc. Nat. Acad. Sci. U.S. 40, 6 (1954).

14. Moore, C. C.: Proc. of the fifth Berkeley Symposium on Mathematical statistics and probality, Vol. II, part. II, p. 447 (1967).

\author{
G. RIDEAU \\ Physique Théorique \\ Institut Henri Poincaré \\ 11, Rue Pierre Curie \\ F-75 Paris Ve
}

